\title{
Matching Dependence-Related Queries in the System Dependence Graph
}

\author{
Xiaoyin Wang ${ }^{1}$, David Lo ${ }^{2}$, Jiefeng Cheng ${ }^{3}$, Lu Zhang ${ }^{1 *}$, Hong Mei ${ }^{1}$, Jeffrey Xu Yu ${ }^{3}$ \\ ${ }^{1}$ Key Laboratory of High Confidence Software Technologies (Peking University), \\ Ministry of Education, Beijing, 100871, China \\ ${ }^{2}$ School of Information Systems, Singapore Management University \\ ${ }^{3}$ The Chinese University of Hong Kong, China \\ \{wangxy06,zhanglu,meih\}@sei.pku.edu.cn, davidlo@smu.edu.sg, \{jfcheng yu\}@se.cuhk.edu.hk
}

\begin{abstract}
In software maintenance and evolution, it is common that developers want to apply a change to a number of similar places. Due to the size and complexity of the code base, it is challenging for developers to locate all the places that need the change. A main challenge in locating the places that need the change is that, these places share certain common dependence conditions but existing code searching techniques can hardly handle dependence relations satisfactorily. In this paper, we propose a technique that enables developers to make queries involving dependence conditions and textual conditions on the system dependence graph of the program. We carried out an empirical evaluation on four searching tasks taken from the development history of two real-world projects. The results of our evaluation indicate that, compared with code-clone detection, our technique is able to locate many required code elements that codeclone detection cannot locate, and compared with text search, our technique is able to effectively reduce false positives without losing any required code elements.
\end{abstract}

\section{Categories and Subject Descriptors}

D.2.7 [Software Engineering]: Distribution, Maintenance and Enhancement

\section{General Terms}

Reliability, Standardization

\section{Keywords}

System Dependence Graph, Code Search, Graph Indexing

\section{INTRODUCTION}

In software development, developers often need to apply one change to a number of similar places in the code. Such a situation occurs typically when developers want to make a programming style change or want to change the environment (e.g., the database system and the user interface) of a software system. For example, when a developer wants to extract a common code pattern to form a new method, he or she should try to locate all the instances of the code pattern and change them to invocations of the new method.

${ }^{*}$ Corresponding author

Permission to make digital or hard copies of all or part of this work for personal or classroom use is granted without fee provided that copies are not made or distributed for profit or commercial advantage and that copies bear this notice and the full citation on the first page. To copy otherwise, to republish, to post on servers or to redistribute to lists, requires prior specific permission and/or a fee.

ASE'10, September 20-24, 2010, Antwerp, Belgium.

Copyright 2010 ACM 978-1-4503-0116-9/10/09 ...\$10.00.
It is usually easy for a developer to locate one or a few places for change, but it is challenging for he or she to locate all the places that require the same change. Since the code base of a modern software application is large and usually many developers have been coding for the application, it may be infeasible for the developer to remember all the similar places without missing any one. Thus, a tool that helps developers locate all the similar places requiring the same change may save much development time.

One possible way to achieve the preceding purpose is to use techniques for code-clone detection. Techniques for code-clone detection are able to detect all the places in the code that are similar to the known places that need change. However, techniques for code-clone detection rely on a pre-defined uniform similarity metric to measure the similarity between two places. But the similarity in the preceding problem is actually some common characteristics that similar code elements should have, and a different change may imply different common characteristics. For instance, as shown in Example 1 in Section 2, the two code elements are "similar" for only the change at hand, but should not be similar in any technique for code-clone detection. Another possible way to achieve the preceding purpose is to use text search. In a typical text-search tool, a developer can represent the common characteristics as a regular expression and search in the code for matched code elements. However, text-search tools cannot always represent common characteristics as regular expressions. For instance, as shown in Example 2 in Section 2, the common characteristics of the need-to-change code elements are based on control and data dependence relationships, and cannot be represented as a regular expression.

In this paper, we propose a novel technique to locating code elements with common dependence-related properties in source code. The basic idea of our technique is as follows. Our technique allows a developer to write a query using a language named the Dependence Query Language (DQL) (presented in Section 4.1). DQL allows a developer to describe dependence properties on top of textual properties. Then, our technique transforms the query into a series of graph reachability patterns and matches them in the System Dependence Graph (SDG) of the source code using a fast algorithm for graph-pattern matching [3]. We empirically evaluated our technique using four searching tasks in two real-world projects. Our empirical results indicate that our technique is more effective than both code-clone detection and text search for these searching tasks.

As a general approach that locates code elements with common dependence-related properties, our technique can be applied to a number of software maintenance tasks. For example, provided with a list of error patterns, our technique can be incorporated in a bug finding tool to locate the error patterns. Another scenario is that when developers want to perform refactorings, they can use our DQL (perhaps with some extensions) to define the code places to be 
changed in the refactorings. Our technique can also be adapted to solve to some specific problems, such as locating the strings flowing to the GUI in software internationalization [19], or locating the variables whose values come from or are compared with sensitive data in security analysis.

This paper makes the following main contributions:

- A demonstration of challenges in locating all the code elements requiring the same change.

- A query language (i.e., DQL) that allows developers to describe dependence-related properties involving multiple program points.

- A technique that locates code elements with certain dependencerelated properties via graph matching.

- An empirical evaluation demonstrating the effectiveness and the cost of our technique.

\section{EXAMPLES}

In this section, we present two examples to demonstrate the challenges in locating all the need-to-change code elements when developers want to apply a change to similar code elements.

The first example comes from code changes made to the expat ${ }^{1}$ project (cvs version 2002-05-17). In the code changes, the developers want to fix some memory leaks that may happen when a call of the malloc function fails. One of the changes is as below.

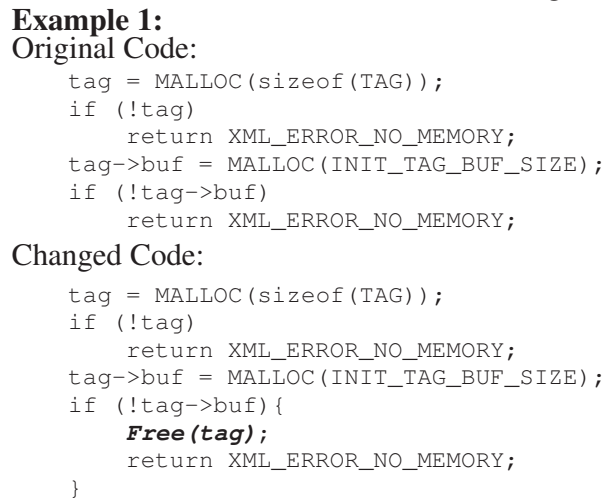

From the preceding two code portions, we can see that the developers want to find cases where a field (i.e., tag->buf in the code) of a struct variable (i.e., tag in the code) is initialized after the struct variable is initialized. The aim is to add a free function when the initialization of the field is failed to avoid memory leak. The key characteristic is two calls of the malloc function: One call is to acquire memory for the entire struct variable and the other call is to acquire memory for a field of the struct variable. As the name of the struct variable and the name of the field may vary in other places, it is difficult to find these places with codeclone detection. Furthermore, in text search, as it is impossible to express the constraint between the struct variable and the field, we cannot use this constraint to confine the searching results. The following gives another location exhibiting the memory leak pattern mentioned above:

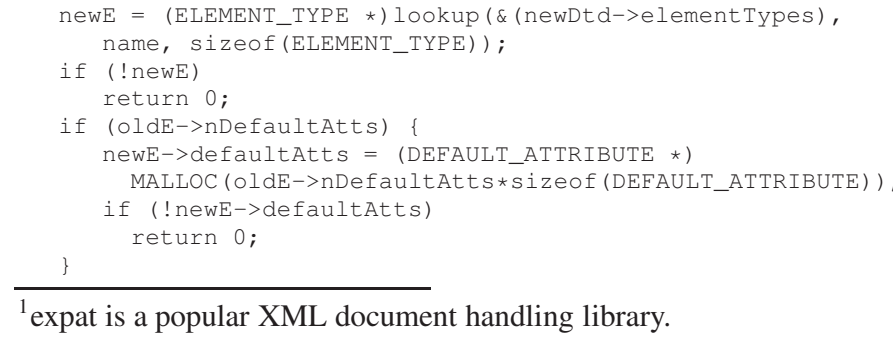

$\overline{1}$ expat is a popular XML document handling library.

In the preceding code portion, the name of the struct variable is newE and the name of the filed is newE->defaultAtts. Furthermore, this code portion only depicts one call of malloc, and the other call of malloc is inside the implementation of the lookup function. To make the situation even worse, there is an extra ifstructure (i.e., if $(o l d E->n D e f a u l t A t t s))$ in this code portion. This example demonstrates that neither code-clone detection nor text search allows developers to express some important constraints when searching for need-to-change code elements.

The second example is from code changes in the gpsbabel $^{2}$ project (cvs version 2004-10-27). In these changes, the developers try to find copying operations between two arrays of type UC in the form of loops. The aim is to replace each such copying operation with a call of a specially defined function (i.e., arraycopy). The following two code portions depict the code before and after such a change. The code in the italicized parts highlights the change.

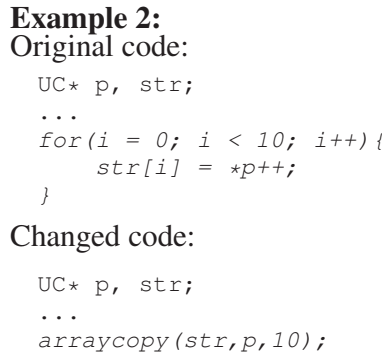

The key characteristics is a series of assignments between elements in two arrays and each element is of type UC. In this example, the main challenge is to express both the following constraints: 1) the constraint between the loop structure and the content inside the loop structure, and 2) the constraint on the type of the elements in the two arrays. Neither code-clone detection nor text search allows developers to express both constraints in one search.

From the preceding two examples, we have the following observations. First, when locating similar code elements for change, developers often need to express searching conditions as dependencerelated properties. Code-clone detection may be ineffective, since code elements satisfying such a searching condition may not look similar. Text search may be ineffective, since such a search condition contains dependence properties besides textual properties. Second, dependence properties described in such a search condition may include both control dependencies and data dependencies.

Based on the preceding observations, we propose a new technique to locating code elements with common dependence-related properties in source code. Our technique allows developers to express combinations of textual conditions, data dependence conditions, and control dependence conditions in individual queries. To deal with the dependence conditions, our technique transforms the queries to graph reachability patterns and matches the patterns in the System Dependence Graph (SDG) of the source code.

\section{BACKGROUND}

\subsection{System Dependence Graph}

The system dependence graph (SDG) is a graph that describes the dependence relationships between program points in the source code [16]. In an SDG generated by CodeSurfer ${ }^{3}$, each node corresponds to a program point in the code. There are 33 types of program points in total in an SDG generated by CodeSurfer. The

\footnotetext{
${ }^{2}$ gpsbabel is a processing system of GPS information

${ }^{3}$ CodeSurfer is a static program analyzer and it can extract a system dependence graph from source code. CodeSurfer can be obtained from http://www.grammatech.com/products/codesurfer.
} 


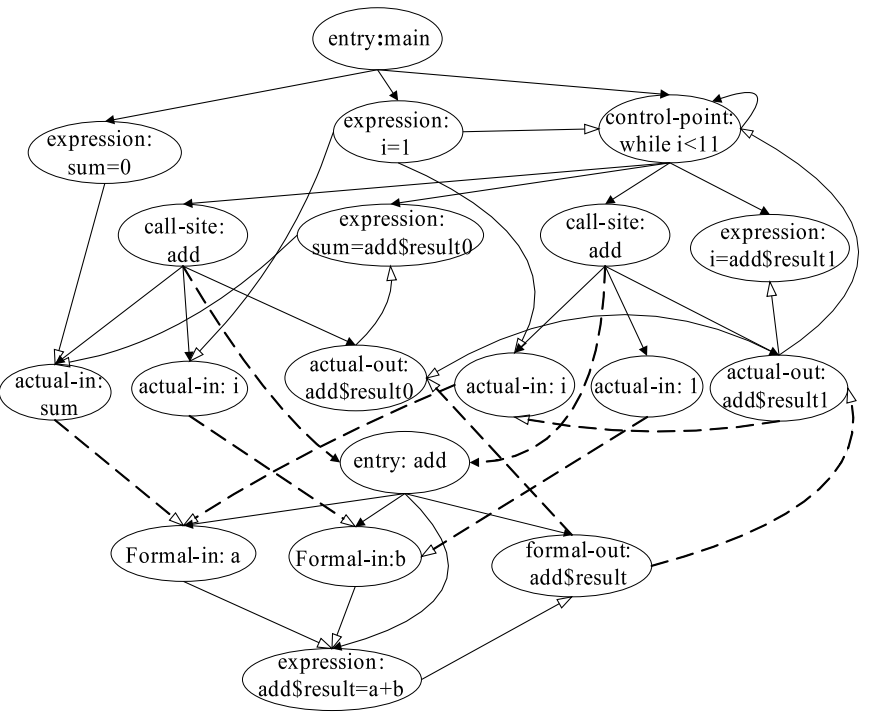

Figure 1: An example system dependence graph

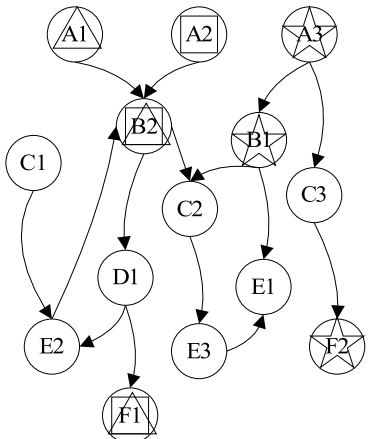

(a)

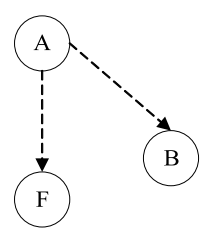

(b)
Figure 2: (a) Example Graph and (b) Query

edges in an SDG correspond to the dependence relationships between program points. There are two kinds of dependence relationships: data dependence relationships and control dependence relationships. Table 1 depicts the most commonly used types of program points in SDGs. In our current technique, we only consider searching for the structures consisting of program points of these most commonly used types. However, it is possible to extend our technique to search for other program points by extending our dependence query language (See Section 4.1) and the query transformation rules (See Section 4.3). Note that program points of other types may be intermediate nodes in various dependence relations between the common program points in the SDG.

Given a C program, CodeSurfer can extract an SDG automatically during the compilation of the program. For example, for the following program, CodeSurfer provides an SDG in Figure 1. In the figure, each node is labeled with its program-point type, and we also provide the textual representation of each node. Hollow arrows refer to data dependencies and solid arrows refer to control dependencies. Solid lines refer to intra-procedure dependencies and dotted lines refer to inter-procedure dependencies.

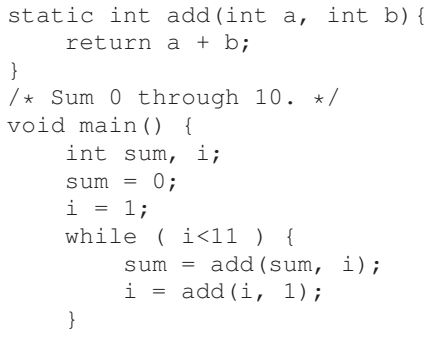

Table 1: Commonly used types of program points

\begin{tabular}{|c|l|}
\hline Program Point Type & Description \\
\hline expression & value assignment or value return \\
\hline control point & $\begin{array}{l}\text { any kind of branch condition } \\
\text { (e.g., if, for, while, ...) }\end{array}$ \\
\hline call site & function invocation \\
\hline actual in & actual argument in a function invocation \\
\hline actual out & returned value of a function invocation \\
\hline declaration & declaration of a variable \\
\hline entry & entry of a function \\
\hline
\end{tabular}

\subsection{Graph Reachablity Indexing and Querying}

As stated in Section 1, our technique transforms a dependence related query to a graph reachability pattern. The problem of matching such a pattern in a large graph is computationally expensive. First, the reachability calculation between all pairs of nodes in a graph has a complexity cubic to the size of the graph. Second, a naive algorithm for matching a graph pattern in a graph has a complexity of $N^{x}$, where $N$ is the size of the graph, and $x$ is the size of the graph pattern.

Fast graph reachability indexing and querying is a new achievement in the area of graph databases [3], which makes graph reachability pattern matching on large graphs computationally acceptable. A graph reachablity indexing algorithm takes a labeled directed graph as input. In a labeled graph, each node has one and only one label from a known label set. The algorithm indexes all the reachability relationships between node labels. With the indices, one can query and obtain all the instances of a reachability pattern (represented as a query graph). For example, for the graph in Figure 2(a) containing the label set $\{\mathrm{A}, \mathrm{B}, \mathrm{C}, \mathrm{D}, \mathrm{E}, \mathrm{F}\}$, a query in Figure 2(b) can be made. To distinguish nodes labeled with the same label, in Figure 2(a), we add numbers to the end of the label. For example, A1, A2, and A3 are three nodes labeled with A. The query in Figure 2(b) denotes sub-graphs having the following conditions: First, in such a sub-graph, there is at least one node labeled with A, one node labeled with B, and one node labeled with F. Second, in such a sub-graph, it is reachable from the node labeled with A to either the node labeled with B or the node labeled with F. Note that edges between nodes in the query graph represent reachability relations instead of immediate connections. The three matched instances (A1, B2, F1; A2, B2, F1; A3, B1, F2) are marked with triangles, squares, and stars, respectively.

\section{APPROACH}

To help developers perform dependence-related queries, we design a language, named the dependence query language (DQL), for developers to describe the common characteristics of target code elements. Section 4.1 presents the details of DQL. With a query written in DQL, our technique searches for code elements that satisfy the common characteristics described in the query using the following four steps. First, we extract the System Dependence Graph (SDG) from the source code using CodeSurfer. Second, we transform dependence conditions in the query to a series of graph reachability patterns. Third, we search in the SDG to locate subgraphs matching the graph reachability patterns. Finally, we use the textual constraints in the query to filter the located sub-graphs and trace back to code elements in the source code. Sections 4.2 to 4.5 presents the details of the preceding four steps, respectively.

\subsection{Dependence Query Language}

A query in our Dependence Query Language has four parts, formally presented as below.

Query $\rightarrow$ QueryDeclaration ; NodeDescriptions ; RelationDescriptions; Wanted 
The QueryDeclaration part is formally defined below.

QueryDeclaration $\rightarrow$ QueryDeclaration, NodeDeclaration I NodeDeclaration

NodeDeclaration $\rightarrow$ Types Identifier

Identifier $\rightarrow[\mathrm{A}-\mathrm{Z}]$

Types $\rightarrow$ Types / Type | Type

Type $\rightarrow$ function | variable | assignment | declaration | control-point

This part allows a developer to declare a list of program points involved in the search. Besides an identifier, the developer must give each program point a list of types. If one program point has more than one type, the meaning is to declare a program point whose actual type can be any one of the listed types. DQL supports five types of program points: function, variable, assignment, declaration, and control-point. These five types are abstraction of the types of program points in SDG. We describe the mapping between types in DQL and types in SDG in Section 4.3. The function type corresponds to any invocations of functions. The variable type corresponds to single variables. The assignment type corresponds to assignments or returns of invocations passing the return values ${ }^{4}$. The declaration type corresponds to declarations of variables. The control-point type corresponds to all branch conditions.

The NodeDescriptions part is formally defined as below.

NodeDescriptions $\rightarrow$ NodeDescriptions NodeDescription I NodeDescription

NodeDescription $\rightarrow$ Identifier Conditions

Conditions $\rightarrow$ Conditions or Condition | Condition

Condition $\rightarrow$ not UnitCondition | UnitCondition

UnitCondition $\rightarrow$ contains String

UnitCondition $\rightarrow$ infile String

UnitCondition $\rightarrow$ infunction String

UnitCondition $\rightarrow$ at Line Number

UnitCondition $\rightarrow$ declareType String

UnitCondition $\rightarrow$ declareType Native

UnitCondition $\rightarrow$ controlType CTYPE

CTYPE $\rightarrow$ for | while | switch | if

In the preceding definition, "Number" corresponds to a positive number. "String" corresponds to a character sequence inside quotation marks, "Native" corresponds to the set of built-in types of the C language, including "char", "float", etc.

This part allows a developer to describe the properties related to a single program point. For each program point, a developer can add three kinds of properties as conditions. First, a developer can use contains to demand the textual representation of a program point to have a particular substring. Second, a developer can describe the location of a program point by providing its exact line number, or by providing the substrings contained in the textual representation of its enclosing files or functions. Third, for certain types of program points, a developer can also describe the declared type or the control type of a given program point. A developer can also add not or or to the conditions for a program point. Note that our DQL does not support the disjunction of conditions of the relationships between different program points. In such a case, a developer needs to generate more than one queries.

The RelationDescriptions part is formally defined as below. RelationDescriptions $\rightarrow$ RelationDescriptions,

RelationDescription | RelationDescription RelationDescription $\rightarrow$ Identifier op Identifier

op $\rightarrow$ dependop | textualop | structuralop

dependop $\rightarrow$ oneStep subDependop

subDependop $\rightarrow$ dataDepends | controls | calls

textualop $\rightarrow$ textual contains

structuralop $\rightarrow$ isfieldof

structuralop $\rightarrow$ iselemento

This part allows a developer to describe three kinds of dependence relationships between program points: dataDepends, controls and calls. Specifically, "A dataDepends B" means that A is

\footnotetext{
${ }^{4}$ We merge these two kinds of elements for simplicity, a developer can demand an assignment to be a return statement using a textual condition.
}

data dependent on $\mathrm{B}$; "A controls $\mathrm{B}$ " means that $\mathrm{B}$ is control dependent on $\mathrm{A}$; and "A calls $\mathrm{B}$ " means that there is a chain of function invocations from A to B. A developer can also use modifier oneStep before a relationship to demand that the dependence must happen in one step in the SDG.

Our DQL also allows a developer to add textual conditions between the textual representations of two program points. Specifically, "A textual contains B" means that the textual representation of $\mathrm{B}$ is a substring of the textual representation of A.

Sometimes program points may have structural relationships, such as the relationship between a struct variable and its fields or the relationship between an array variable and its elements. So DQL also supports structural conditions. In particular, "A isFieldOf B" means that $\mathrm{A}$ is a field of struct variable $\mathrm{B}$, and " $\mathrm{A}$ isElement Of $\mathrm{B}$ " means that A is an element of array variable B. For example, "a.b isFieldOf a" holds; "a[b] isElementOf a" holds.

The Wanted part is formally defined as below. This part allows a developer to indicate which program points in the query are the actual target.

Wanted $\rightarrow$ want Identifiers

Identifiers $\rightarrow$ Identifiers, Identifier | Identifier

Note that the syntax of DQL allows developers to write meaningless queries (e.g., defining a function A with control type if, or defining function A calls variable B). The meaningless queries are checked and deemed as errors when we transform the queries to graph patterns (see Section 4.3.2).

We next give a DQL query for Example 1 in Section 2. In the example, since the only place requiring change is the initialization of the field of the struct variable, the developer can set only the program points corresponding to the initialization of the field as the targets. In particular, a query for this example is as below:

function $A$, function $B$, variable $C$, variable $D ; A$ contains "malloc" or contains "realloc", B contains "malloc" or contains "relloc"; $C$ dataDepends A, D dataDepends B, Conestep dataDepends $D, C$ isField $O f D$; want $C$

In the query, $A$ corresponds to the initialization of the struct variable, $B$ corresponds to the initialization of the field, $C$ corresponds to the field, and $D$ corresponds to the struct variable. We use dataDepends to describe the relationship from the initialization of the variable to the variable itself. We further use onestep dataDepends and isFieldOf to describe the relationship between a struct variable and its fields.

\subsection{SDG Extraction}

In our technique, we obtain the system dependence graph (SDG) as follows. First, we use CodeSurfer to generate an initial SDG from the code. Second, we check each node to see whether the node is a program point of one of the types listed in Table 1. For each such node, we extract its type (e.g., actual-in, call-site, etc.), textual presentation, and location in the code, and label the node with its type. For other nodes, we also label each node with its type but do not extract further information. We keep these nodes in the SDG to transit dependence relationships between nodes of the most commonly used types. Third, we extract all the edges between nodes and label them as control dependence or data dependence. Here we do not differentiate inter-procedure dependence and intraprocedure dependence, since a developer usually does not know whether the target he or she is searching for is in a function or scattered in different functions. Fourth, we give each node in the SDG a unique number as its identifier.

\subsection{Query Transformation}

To use graph reachability querying to search in the SDG, we need to transform a query described in DQL into one or more queries for 
graph reachability querying. First, as graph reachability querying accepts only query graphs, we need to transform queries in DQL into query graphs. Second, as a query graph in graph querying allows only the conjunction of label conditions and reachability conditions, we need to split a query with disjunctions of conditions in DQL into several query graphs. Third, for conditions (such as structural relationships, textual relationships, program-point properties described in the NodeDescriptions part, and the oneStep conditions) supported by DQL but not supported by graph reachability querying, we do not transform them into query graphs but use them to filter the results of graph reachability querying. We present the details of filtering in Section 4.5.

\subsubsection{Splitting Queries}

In a query written in DQL, there are two places that may contain disjunctions of conditions, and the conditions in both places are conditions for single program points. The first place is the QueryDeclaration part, where a program point may have multiple possible types. The second place is the NodeDescriptions part, where the properties of a program point may be the disjunction of several conditions. To deal with the disjunctions of conditions, we split the query into the disjunction of a series of sub-queries, each of which contains only conjunctions of conditions. This process is similar to the process of normalizing a logic expression into the disjunctive normal form. When querying the SDG, we use each conjunctive sub-query to obtain a set of results and use the union of all the result sets as the results of the original query.

For example, for the query described as "function / control-point A, variable B; A contains "abc" or contains "de"; A dataDepends $B$; want $A$ ", we can split the query into the following four subqueries: "function A, variable B; A contains "abc"; A dataDepends $B$; want A", "control-point A, variable B; A contains "abc"; $A$ dataDepends B; want A", "function A, variable B; A contains "de"; $A$ dataDepends $B$; want $A$ ", and "control-point $A$, variable $B ; A$ contains "de"; A dataDepends B; want A". None of the sub-queries includes any disjunctions of conditions.

\subsubsection{Transforming Conjunctive Queries}

For a query containing only conjunctions of conditions, we transform the query into a query graph in the following way. First, we transform each program point in the QueryDeclaration part into a node in the query graph. Second, we do not consider the conditions for filtering (e.g., properties described in the NodeDescriptions part) in query transformation. Third, we transform the type of each program point into the label of the corresponding node, and the relationships between program points into edges between nodes. Note that there is no straightforward one-to-one mapping between the types of program points in DQL and the types in the SDG and there is no straightforward one-to-one mapping between the relationships in DQL and the reachability relationships in the SDG. In the following, we present the details of transforming program-point types and relationships between program points.

Figure 3 depicts the rules for the preceding transformation. Each rule corresponds to the transformation of two program points (including their types) and their relationship described in DQL into the corresponding sub-graph (including node labels) in the query graph. In the figure, hollow arrows denote data dependence and solid arrows denote control dependence. Note that some rules result in adding extra nodes into the query graph.

For brevity, in Figure 3, we merge rules when the relationship in $\mathrm{DQL}$ is the same and the structure of the transformed sub-graph is similar. As a result, we have four generic rules in Figure 3, and in each rule, a program point in DQL may have more than one type and a node in the transformed sub-graph may have more than one

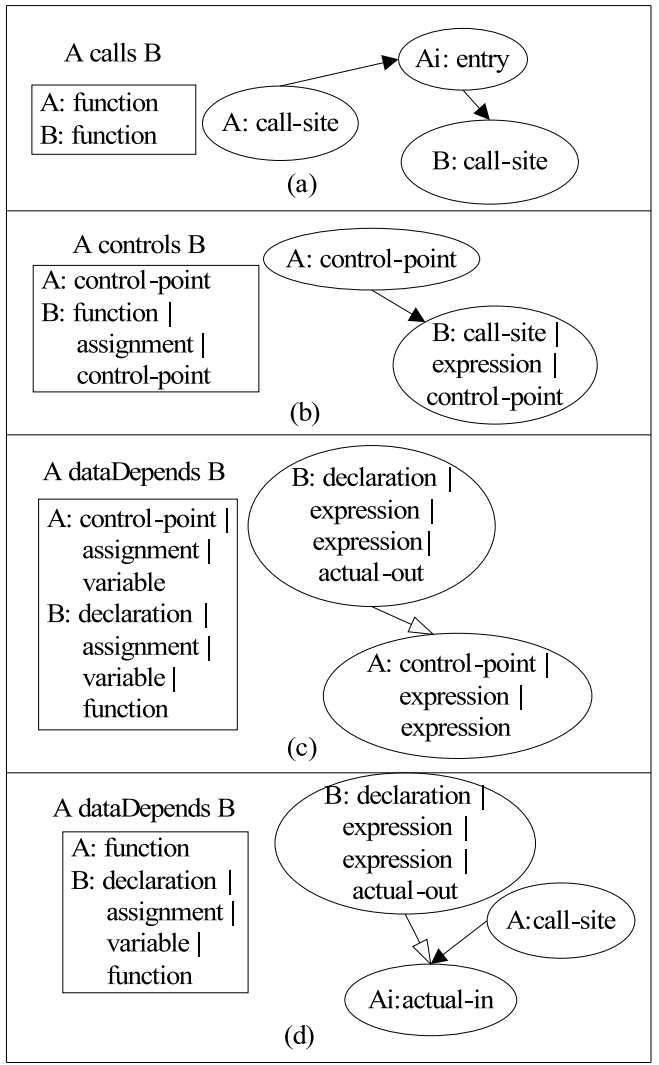

Figure 3: Rules for dependence relation descriptions

label. In such a case, we use "l" to separate the program-point types and the node labels. Details of the four generic rules are as below.

- $\boldsymbol{A}$ calls $\boldsymbol{B}$. According to the SDG generated by CodeSurfer, such a situation requires that $B$ should be control dependent on the entry of $A$ and the entry of $A$ should be control dependent on the call-site of $A$. So we add two control dependence edges and an intermediate entry node from $A$ to $B$ (Figure 3(a)). Both $A$ and $B$ should be functions. The newly added node should have an identifier not used by other nodes, and we use $A_{i}$ to indicate that it is the $\mathrm{i}^{\text {th }}$ added node.

- $\boldsymbol{A}$ controls $\boldsymbol{B}$. We transform this relationship to a control dependence edge from $A$ to $B$ (Figure 3(b)). $A$ should be a control-point, and $B$ can be an assignment, a function call or another control-point. Furthermore, if $B$ is a function call, we need to transform the function type into the call-site label. As neither declarations nor references of variables are executable, we do not allow $B$ to be a declaration or a variable. We transform the assignment type into the expression label because the SDG generated by CodeSurfer uses an $e x$ pression to represent an assignment.

- $A$ dataDepends $B$ and $\boldsymbol{A}$ is not a function. We transform this relationship to a data dependence edge from $B$ to $A$ (Figure 3(c)). $A$ can be a control-point, an assignment or a variable. As a declaration cannot be data dependent on anything, we do not allow the type of $A$ to be declaration. $B$ can be of any types except for control-point (CodeSurfer treats an assignment like " $\mathrm{x}=\mathrm{a}>\mathrm{b}$ " as an expression rather than a control point). Two things need further explanation in Figure 3(c). First, when one program point is data dependent on another program point of the function type, the label for the node corresponding to the program point of the function type should be actual-out. This is because in the SDG generated by CodeSurfer, data dependence to a function is denoted 
as data dependence to the actual-out corresponding to the function call. Note that the dependence between an call-site program point and its corresponding actual-out is a control dependence. Second, we transform variable into expression because the SDG generated by CodeSurfer uses an expression to represent an variable.

- $A$ dataDepends $B$ and $A$ is a function. In this case, one of the $A$ 's actual argument should be data dependent on another program point. Furthermore, the dependencies between a call-site and its corresponding actual arguments are control dependencies. So we have the the rule as in Figure 3(d).

Since program points of type function may be transformed to nodes with different labels in different rules. A node may have conflicting labels. Possible conflicting labels are call-site and actualout. In such a case, we split the node to two, and add a control dependence edge from the call-site node to the actual-out node.

If no rule in Figure 3 is able to transform a dependence relationship described in a query, we deem that there is an error in the query. For example, if $A$ is declared as a function and B is declared as a control-point, the dependence relationship described as "A calls $B$ " will result in an error.

\subsection{Graph Querying}

As graph reachability indexing and querying requires only one type of edges in both the query graph and the graph for indexing, we cannot use the query graphs to query the SDG. To enable the query, we divide either the query graph or the SDG to two partial graphs. In each partial graph, the nodes are the same with the original graph but we keep only one kind of edges. Thus, after the two partial SDGs are indexed, we perform two queries using two partial query graphs on their corresponding partial SDGs.

After we obtain the two lists of results, we need to concatenate the results. We mark a list of split nodes in the query graph when doing the graph division. A split node is a node that has related edges in both partial query graphs. For each result $R 1$ in the results for the control dependence query and each result $R 2$ in the results for the data dependence query, we check whether we can merge all the split nodes 5 . If all the split nodes can be merged, we merge the two results and put the merged result into the set of final results.

\subsection{Result Filtering and Generation}

After we obtain the results of graph querying, we further use the conditions that are in the original conjunctive query but not transformed into the query graph to filter the results. For each result, we check three categories of conditions. First, we check each node with its corresponding program point in the query. If any property of the program point is not satisfied, we discard the result. Second, we verify the textual and structural conditions between program points. That is to say, if any two program points do not satisfy any conditions described for them, we discard the result. Third, we verify the oneStep conditions between program points. We examine whether the dependence relationship between two program points is one step by verifying whether there is an corresponding (control or data) edge between the two corresponding nodes in the SDG. For the calls relationships, as such a relationship is transformed to multiple edges in the query graph, we examine whether all these edges are one step.

Finally, as a result after filtering may contain several program points, we recommend the developer with only the program points corresponding to the wanted part. If the developer wants to examine the reason of our recommendation, we allows the developer to expand the result and see all the involved program points.

\footnotetext{
${ }^{5}$ Two nodes in two results can be merged, if the two nodes correspond to a same node in the original SDG graph.
}

\section{OPTIMIZATION}

In our technique described in Section 4, we use only the types of program points as labels of nodes in the SDG and the query graph. Therefore, we may have a huge number of results when querying the SDG, and we need to filter the results using the other conditions described in the query. In some cases, the filtering may become unreasonably time consuming (e.g., more than 24 hours) due to the huge number of results in SDG querying.

To solve the problem, we resort to pre-filtering. The basic idea of pre-filtering is to move the filtering of program-point properties (described in the NodeDescriptions part of a query) before the graph querying step. Given an SDG (denoted as $G$ ) and a conjunctive query (denoted as $Q$ ), we transform $Q$ to a query graph (denoted as $Q G$ ). Based on $G, Q$, and $Q G$, our pre-filtering technique works as follows.

For each node (denoted as $N$ ) in $Q G$, we assign the node a unique label (denoted as $L$ ) and we further find all the nodes in $G$ that match both the type and the properties of $N$. Note that an extra node added into $Q G$ during query transformation has only a type but no other properties. Then, we label all these nodes in $G$ as $L$. The aim of this change of labels is to constrain the matched nodes of $N$ in $G$ during graph querying. In some cases, one node (denoted as $O$ ) in $G$ may match several different nodes in $Q G$. Thus, there is a conflict of label change, since we cannot assign a node in $G$ more than one label. To solve the conflict, we add one or more parallel nodes into $G$.

For the ease of presentation, let us assume that a node (denoted as $O$ ) in $G$ matched two different nodes (denoted as $N 1$ and $N 2$ ) in $Q G$ and the labels of $N 1$ and $N 2$ are $L 1$ and $L 2$, respectively. Thus, we add a new node (denoted as $P$ ) into $G$, such that $P$ has the same neighboring structure as $O$. Specifically, for each node from which there is an edge to $O$, there is also an edge to $P$ of the same kind (control or data dependence), and for each node to which $O$ has an edge, $P$ also has an edge to it of the same kind. Therefore, the new node $P$ has the same reachability property with $O$ and adding $P$ to $G$ would not affect reachability relationships between any other nodes in $G$. We assign the label of $O$ as $L 1$ and the label of $P$ as $L 2$. Thus, during graph querying, $N 1$ can match $O$ but not $P$ in $G$, and $N 2$ can match $P$ but not $O$ in $G$.

Due to added parallel nodes, it is possible that our graph querying algorithm returns a result with two or more nodes corresponding to one original node. In such a result, one node in $G$ is actually matched to two or more nodes in the query graph. As we do not allow this kind of matches, we filter out the matched sub-graphs that contains two or more nodes parallel with each other.

Through pre-filtering, we obtain much less results from graph querying, and we need to filter the results of graph querying with only conditions of relationships between program points and the oneStep conditions. Our pre-filtering can save much time in filtering, but we need to re-index the SDG when a new query comes. However, the time for indexing is much shorter than that for result filtering without pre-filtering.

\section{EMPIRICAL EVALUATION}

To evaluate our technique, we applied our technique on four searching tasks in four versions of two different real-world open source C projects: expat and gpsbabel. Table 2 depicts the detailed information of the four versions of the two projects used in our evaluation. To make it easier to generalize the findings of our evaluation, we chose two of the most downloaded $\mathrm{C}$ projects in Souceforge $^{6}$, and the two projects are of different sizes and from different domains.

\footnotetext{
${ }^{6}$ http://sourceforge.net/
} 
Table 2: Subjects used in our evaluation

\begin{tabular}{|c|c|c|l|}
\hline Project Name & Description & Version & Size (KLOC) \\
\hline expat & xml handle library & $2002-05-17$ & 13 \\
\cline { 3 - 4 } & & $2002-05-22$ & 13 \\
\hline gpsbabel & \multirow{2}{*}{ GPS toolkit } & $2004-10-27$ & 50 \\
\cline { 3 - 4 } & & $2005-03-21$ & 54 \\
\hline
\end{tabular}

To make the four searching tasks close to real scenarios that developers may face, we extracted the four searching tasks from the historical versions of the two projects. In particular, we browsed the historical versions of the two projects, and when we found multiple modifications in one version over its previous version, we checked the comments submitted with the modifications. If the comments imply the application of one change to a number of places, we deemed that the developers need to perform a searching task to apply one change to multiple places.

For each of the four tasks, we built a DQL query based on one changed place that has comments implying the change in multiple places. Section 6.1 presents the results of applying our technique on the four tasks. For comparison, we also applied a technique based on code-clone detection and a technique based on text search to the same tasks. For the technique based on code-clone detection, we applied a code-clone detector named DECKARD [10] to each of the four versions. We then checked each clone group returned by DECKARD, and we deemed the clone group containing the largest number of places required by the searching task as the results of the technique based on code-clone detection. For the technique based on text search, we built several possible text queries and recorded the results using each text query.

\subsection{Results for the Searching Tasks}

\subsubsection{Task One}

The first searching task is actually Example 1 in Section 2. It is from the expat project version 2002-05-17. The comment of the code change is "Be more careful about failed MALLOC() and REALLOC() calls. This avoids a number of potential memory leaks". Example code of the change is shown in Section 2. The query we used has been introduced at the end of Section 4.1.

In this task, the developers actually changed 2 places (i.e., targets) in the code. Our technique finds both of the 2 places, but our technique also finds 36 other places, which are false positives. By contrast, it is not surprising that the technique based on code-clone detection does not find a clone group containing the 2 targets. As shown in Section 2, the two places do not look similar in the sense of code cloning. For text search, the only reasonable way is to search for "malloc" and "realloc" in the code. Since the two related "malloc" or "realloc" may be in different functions (and in one of the 2 changed places, they indeed are in different functions), it is not possible to search two continuous "malloc" or "realloc" using regular expression searching. Text search finds 33 places in the code containing "malloc" or "realloc". As this task requires two related "malloc" or "realloc", a developer may need to check each pair of the 33 places (528 pairs) to know whether the pair forms a target required by the task and find the 2 correct pairs. Note that one of the 2 targets actually involves two invocations of "malloc()" in two different functions. Not checking this pair of places with "malloc" may miss one target.

\subsubsection{Task Two}

The second searching task is from the expat project version 200205-22. The comment of the code change is "Use "NULL" instead of " $O$ " for NULL pointers. Compare pointers $==N U L L$ or $!=N U L L$ instead of using the implicit point-to-int conversion". This comment clearly reflects the purpose of the developers (i.e., changing comparisons of pointers with 0 to comparisons of pointers with NULL). Note that compiler does not catch these cases as warnings.

As there are a large number of control points in the program, the essence of this searching task is actually to find all the comparisons of pointers. Therefore, we have the query below.

declaration A, control-point B; A not declareType Native; $B$ oneStep dataDepends $A$; want $B$

This query actually finds all the comparisons of variables of nonnative types. In the query, $A$ corresponds to a declaration of a nonnative variable, while $B$ corresponds to the control point that involves $A$. The dependence from $B$ to $A$ is one step because $A$ should directly declares the variable involved in $C$. Note that the query is not very specific to the task and may be refined to get better results.

In this task, the developers actually changed 8 places (i.e., targets) in the code. Our technique finds all the 8 targets together with 200 other places. Among the 200 places, 22 are false positives. The other 178 of the 200 places are also comparisons between pointer variables and 0 . Thus, these code elements should also be changed. It is probable that these code elements are too difficult to be found so that the developers did not change them. By contrast, the technique based on code-clone detection still does not find any of the 8 targets. This is also not surprising because the code-clone detector would not deem comparisons of different pointers as cloning. For text search, one possible way is to search for the string " $==0$ " and "!=0" in the code. This search finds only 2 of the 8 targets together with 70 false positives and 10 comparisons between pointers and 0 . This search misses 6 targets due to implicit comparisons with " 0 " when a pointer variable is used as a condition variable (e.g., p in if $(p)$ ). Another possible way of using text search is to search for strings like "for", "while", "switch" and "if" in the code, since all the comparisons should appear in these control structures. The second text search finds all the 8 targets, with 829 other places. These 829 places also include the 178 comparisons between pointers and 0 , and 651 false positives.

\subsubsection{Task Three}

The third searching task is actually Example 2 in Section 2 and is from the gpsbabel project version 2004-10-27. The comment of the code change is "Aggressively replace open-coded strncpy for space padded strings in various waypoint send functions". The submitted code changes demonstrate that the developers try to find all the code elements that are in "waypoint send" functions (a list of functions whose names end with "send") and are like the italicized part in the following code portion.

$\mathrm{UC} * \mathrm{p}$, str; $\ldots$

for $(i=0 ; i<10 ; i++)\}$

str $[i]=* p++;$

The preceding code structure has two dependence relationships. First, there is a loop (either a for loop or a while loop). Second, inside the loop, there is an assignment and the assignment data depends on a declaration that declares a variable with type $U C \star$ used in the assignment. Third, according to the code-submission comment, the loop and the assignment should be in a function whose name contains "send". Therefore, we have the query as below.

declaration A, control-point B, assignment $C$; A declareType $U C^{*}$, $B$ controlType for or controlType while, B inFunction "send", $C$ inFunction "send"; B onestep controls $C$, C oneStep dataDepends A; want $B$

In the preceding query, $\mathrm{A}, \mathrm{B}$ and $\mathrm{C}$ correspond to three program points in the example. A should be a variable declaration and the type of the variable is " $\mathrm{UC}^{*}$ ", and the node B should be a loop and its control type is "for" or "while". The dependence from C to A is one step because A should directly declares C. The dependence from $\mathrm{B}$ to $\mathrm{C}$ should be one step because the assignment should 
be executed in each iteration of the loop to copy an array. Thus the assignment should be directly controlled by the loop control point. B is wanted because the developer wants to locate only the for / while loops. B should be in a function whose name contains "send". Note that our technique currently does not support searching only in the functions whose name ends with "send".

In this task, there are actually 37 targets in the code. Our technique finds all the 37 targets together with 25 false positives. By contrast, the technique based on code-clone detection finds 36 targets with 23 false positives. Unlike the previous two tasks, the targets in this task share a common loop structure that can be caught by the code-clone detector. The one target that code-clone detection fails to find is also a for loop. But there are several other statements in the for loop, so that the similarity between the lost target and the other targets drops below the threshold. Our technique brings in 2 extra false positives, because the conditions in our query is not as strict as the threshold in code clone detection. For text search, one possible way is to search for "for" and "while" in the code. This search finds all the 39 targets, because all the targets involve a for loop or a while loop. But this search also finds 297 false positives, because there are many for or while loops used for other purposes. Another possible way is to search for UC* in the code and find all the variables of type UC $\star$. Thus a developer needs to further locate all the references to the these variables to check the assignments of these variables in for or while loops. In total, the developer needs to check more than 2000 references to the variables with type $\mathrm{UC} \star$ in the code.

\subsubsection{Task Four}

The fourth searching task is from the gpsbabel project version 2005-03-21. The comment of the code change is "Call waypt_new instead of explicit calloc to prepare for external alt invalid indicator". In the submitted changes, the aim is to change all xcalloc () function calls that initialize variables of type waypoint* to calls of a new function (i.e., waypt_new()) that specially initializes waypoint* variables. An example is as below.

waypoint *wpt_tmp;

...

wpt_tmp $=x c a l l o c\left(\right.$ sizeof $\left.\left(* w p t \_t m p\right), 1\right)$;

In the preceding code element, there are two dependence relationships. The first is data dependence between the waypoint *wpt_tmp declaration and the wpt_tmp variable. The second is data dependence between xcalloc () and wpt_tmp. Thus we have the query as below.

declaration $A$, variable $B$, function $C$; A declareType waypoint, C contains "xcalloc"; B oneStep dataDepends A, B oneStep dataDepends $C$; want $C$

In the query, $A$ corresponds to the declaration of a variable with the waypoint * type. $B$ corresponds to the variable with the waypoint* type. $C$ corresponds to the invocation of xcalloc. The dependence between $B$ and $C$ is one step because variable $C$ should be the variable that first holds the return value of xcalloc(). The dependence between $B$ and $A$ is one step because $A$ should directly declares $B$. $C$ is wanted because the developer wants to locate only xcalloc () calls.

In this task, there are actually 19 targets in the code, and our technique finds all the targets together with 3 false positives. By contrast, the technique based on code-clone detection finds 17 targets with 9 false positives. In two of targets (xcalloc () invocations), the argument structure is different from that of other targets, so that the code clone detection technique fails to find them. The extra false positives are xcalloc() invocations that initialize variables with types other than waypoint*. In text search, one possible way is to search for "xcalloc" in the code. This search finds all the 19
Table 3: Execution time of our technique (in seconds)

\begin{tabular}{|l|l|l|l|l|l|l|l|}
\hline Task & $\begin{array}{l}\text { Query } \\
\text { Build }\end{array}$ & $\begin{array}{l}\text { Pre- } \\
\text { filter }\end{array}$ & Index & Query & Concat & $\begin{array}{l}\text { Result } \\
\text { Filter }\end{array}$ & $\begin{array}{l}\text { Result } \\
\text { Merge }\end{array}$ \\
\hline 1 & $<0.1$ & 1.7 & 63.7 & 1.0 & N/A & 2.1 & $<0.1$ \\
\hline 2 & $<0.1$ & 0.9 & 29.4 & $<0.1$ & N/A & 1.1 & N/A \\
\hline 3 & $<0.1$ & 3.3 & 640.0 & 0.2 & 4.2 & 3.6 & $<0.1$ \\
\hline 4 & $<0.1$ & 3.3 & 264.4 & 1.2 & N/A & 0.9 & N/A \\
\hline
\end{tabular}

targets, but concedes 86 false positives. The reason is that there are many other places that invoke xcalloc(). Another possible way is to search for "waypoint" in the code, and further check all the references of variables with type waypoint $\star$. This search returns more than 600 references to variables with type waypoint*.

\subsection{Execution Time}

We also recorded the execution time of each step in our technique for performing each of the four tasks. We did not record the execution time of of text search and code clone detection because both of them have short execution time. Table 3 depicts the results on execution time. We only present the execution time of doing code queries with pre-filtering, because without pre-filtering the code query cannot return results within 24 hours for each of the tasks. Note that we only present the execution time of steps that are involved in performing a query. We do not present the execution time of the preparation steps including compiling the projects, extracting SDGs, and dividing SDGs to two partial SDGs (each one has only one kind of edges). The execution time of the preparation steps are unimportant because once the these steps are performed, the developers can do any number of queries without performing these steps again. Furthermore, the preparation steps are performed together with the project compilation, so that it brings in little extra cost for developers. Actually, the execution time of preparation steps is about two minutes for the tasks from expat and about ten minutes for the tasks from gpsbabel.

In Table 3, there are three things to be noted. First, the execution time of "Query Build" (column 2) is the total execution time of splitting a query to conjunctive queries (if query splitting is required), transforming from conjunctive queries to graphs, and dividing graphs to partial graphs that contain only one kind of edges (if graph division is required). Second, the execution time of "Concat" (column 6) is the execution time of concatenating the results of two partial queries. Since queries for some tasks only contain one kind of dependence relationships, our technique did not perform graph division and concatenation and thus the corresponding execution time for concatenation is not available. Third, the execution time of "Result Merge" (column 8) is the execution time of merging the results of conjunctive sub-queries. Similarly, if a query does not contain disjunctives of conditions and thus result merging is not required, its execution time of result merging is not available.

From this table, we can see that the execution time for the shortest search is around half a minute, and that for the longest search is around 11 minutes. The execution time may be too long for developers to use our technique in an interactive manner. However, as our technique does not require user involvement in the searching process, it is possible for developers to use our technique in an offline way. Therefore, the execution time should not be a big burden for developers.

Furthermore, this table also shows that the most time-consuming step in the query process is graph indexing. Currently, since our technique adds some parallel nodes in the pre-filtering step, our technique needs to re-index the graph when a new query comes. As the number of added nodes is small, it should be possible to develop an incremental indexing algorithm to add more indexing 
information about the added parallel nodes on the basis of the existing indexing information. With such an incremental indexing algorithm, we may further accelerate our technique.

\subsection{Threats to Validity}

The main threat to internal validity in our evaluation is the possible faults in the implementation of the evaluated techniques. To reduce this threat, we acquired the implementation of DECKARD from one author of the tool and used the text-search facility of a mature text editor. Furthermore, we reviewed all the code of the implementation of our technique before conducting the evaluation. In our evaluation, we applied our technique with the technique based on code-clone detection and the text-search technique on four searching tasks. This factor may be a threat to the external validity, as our empirical results may be specific to the used tasks and subjects and thus not generalizable. To reduce this threat, we used different tasks with subjects from different domains. As the searching tasks were recovered from the subjects, the scenarios involved in the tasks may not well represents scenarios in real-world development. This factor may be a threat to the construct validity. To reduce this threat, we used the version history of each subject, and carefully examined the change comments and the changed code to figure out the purpose of the developers for each searching task.

\subsection{Discussion}

According to the results presented in Section 6.1, our technique has a considerable number of false positives in the four searching tasks. The false positives are mainly due to that we used simplified ways to specify search requirements. However, compared with code-clone detection and text search, in which many search requirements cannot be specified, our technique does provide a means to appropriately specify different search requirements. In fact, it is still possible to refine the queries for our technique to further reduce the false positives.

The effort spent on defining an appropriate query for our technique is also a concern. For a developer, both his or her familiarity with our technique and with the software project under search may impact the effort spent on defining queries. In our evaluation, we (who are familiar with our technique but not very familiar with either projects) spent several minutes to define a query for each task. We plan to assign some code searching tasks to real developers in the future to study the effort spent on defining queries.

\section{RELATED WORK}

In essence, our technique searches for code elements satisfying some common dependence-related constraints described with our DQL. Thus, the research most related to our technique is various generic code-search techniques. Generic code-search techniques are code-search techniques that allow users to write different queries for different searching tasks. The main advantage of generic code-search techniques is that users can use them in different searching tasks without changing the underlying algorithm.

The most popular category of generic code-search techniques is text search, including plain text search, regular expression search, and natural language based code search $[6,9]$. The main difference between these techniques and our technique is that, besides textual information, our technique further allows users to describe dependence relationships in the target code elements. Our empirical results indicate that descriptions of dependence relationships help improve the accuracy of code search in many searching tasks.

Another category of generic code-search techniques is based on model checking. By using mode checking, such techniques allow users to describe temporal conditions in queries. For example, Li and Zhou used model checkers to locate the outliers of coding rules, such as finding the place where a call to File.open () is not followed by a call File.close () [12]. Another typical example in this category is a technique recently proposed by Brunel et al. [2]. As a supporting technique for a generic patch-inference tool [1] (which infers and applies common patches for Linux drivers), Brunel et al.'s technique can locate code elements satisfying certain controlflow properties using model checking. Unlike Brunel et al.'s technique, our technique uses graph matching to check which code elements satisfy the required control/data-dependence conditions. In most of the searching tasks discussed in this paper, description of data dependence relationships in queries is essentially helpful. Since data dependence relationships in SDGs may not be described as temporal relationships, model checkers may not be suitable to check these data dependence relationships.

Generic code search can also be based on checking properties acquired at runtime. Martin et al. proposed the Program Query Language (PQL) [14], which allows users to describe a pattern of sequentially executed invocations on objects. For each query, the searching technique based on PQL first statically checks the program to acquire a list of candidate program points that may appear in instances of the pattern. Then the candidate program points are instrumented and the pattern is matched dynamically during the execution of the instrumented program. The differences between PQL-based searching and our technique is as below. First, PQL focuses on only invocations of objects, while our technique can also handle many other program points, such as expressions, control points, etc. Second, PQL matches code patterns dynamically, while our technique matches queries statically.

The main disadvantage of generic code-search techniques is that they may not be sufficiently precise on some particular tasks. Therefore, there are intensive investigations on specialized code-search techniques for some commonly encountered tasks. Specialized codesearch techniques search for code elements with pre-defined patterns. The searching algorithm in a specialized code-search technique typically focuses on just one pre-defined pattern and thus can be more precise, but no specialized code-search techniques can search for a pattern other than the pre-defined patterns.

Code-clone detection techniques $[11,10,5]$ focus on finding similar code segments in the code base of a program. Using codeclone detection, developers can search for code portions with certain properties (that some code portions are known to share). Since developers typically have known some code elements when they want to apply a change to other code elements, code-clone detection may be adopted for this purpose. However, our empirical results indicate the superiorness of our technique over code-clone detection for this purpose. We suspect the essential reason to be that code-clone detection techniques are actually specialized codesearch techniques designed for another purpose.

Slicing techniques help to locate a set of the program points that affect or depend on a given variable [20]. In this sense, it is also a specialized code search technique. Compared with our technique, slicing starts from a known variable, and the query is pre-defined (program points affecting or depending on the variable), while our technique allows flexible queries defined by developers.

Our work is also related to techniques for recommendation of code samples [7, 22], which search for matched code samples in the source code of a number of programs. Compared with our technique, these techniques work on only API-invocation structures and use pre-defined rules, while our work handles both API invocations and other program points, and allows users to describe the characteristics of target code samples in queries.

Bug pattern matching tools provide a list of detectors for pre- 
defined bug patterns, and allow developers to develop detectors of new bug patterns using code managing APIs. Two popular bug pattern matching tools are FindBugs ${ }^{7}[8]$ and PMD $^{8}$ [4]. Compared with these two tools, our technique provides a unified searching technique and does not require developers to develop a detector for each new pattern, which could cost substantial development time.

There are also other pattern matching tools for specialized tasks. Maule et al. proposed a technique to search for code elements that are affected by a given database-schema change [15]. Wang et al. proposed a technique to search for constant string variables that finally goes to the GUI to facilitate software internationalization [19]. In a broader sense, feature-location techniques [21] can be viewed as specialized code-search techniques, because such a technique tries to search for code elements that are related to a given feature. Jungloid-search techniques $[13,17,18]$ can also be viewed as specialized code-search techniques, because such a technique tries to search for a conversion code to transform a source object type to a target object type. Compared with these techniques which use pre-defined queries, our technique is much more flexible and matches any queries written in DQL.

\section{CONCLUSION AND FUTURE WORK}

In this paper, we propose a novel technique that helps developers match dependence-related queries in the system dependence graph. Our technique includes: a query language that allows developers to describe dependence relationships, an algorithm to transform queries to graph reachability patterns, a graph indexing and querying algorithm, a result filter, and an optimizer based on prefiltering. We evaluate our technique on four searching tasks acquired from four versions of two real-world $\mathrm{C}$ projects. The results of our evaluation indicate that our technique is able to locate all the targets within reasonable execution time. Furthermore, our technique outperforms code-clone detection and text search by finding more targets or conceding fewer false positives.

There are several ways to improve or extend our technique. First, we plan to address the threats to validity mentioned in Section 6.3 by studying more versions of more projects and doing some user studies in real-world projects.

Second, our technique reports some false positives. We plan to improve our technique to allow developers to query with more information such as providing the default values of variables. We also plan to handle negative dependence conditions (e.g., A does not depend on B), which our current technique does not support. We believe that with these improvements, developers can define more accurate queries and the false positives can be further reduced.

Third, in our evaluation, we did not study the amount of effort needed to define an appropriate query. We plan to carry out such a study by assigning searching tasks to a number of developers and record the time they spend on defining queries.

Fourth, our pre-filtering requires re-indexing of the system dependence graph when a new query comes. The re-indexing is the most time-consuming part in our technique. As re-indexing in our technique actually faces a small number of added nodes, we plan to investigate incremental re-indexing algorithms that can index only the newly added nodes.

\section{Acknowledgments}

The authors from Peking University are sponsored by the National Basic Research Program of China (973) No. 2009CB320703, the High-Tech Research and Development Program of China (863) No. 2007AA010301 and No. 2006AA01Z156, the Science Fund for

\footnotetext{
${ }^{7}$ http://findbugs.sourceforge.net/

${ }^{8} \mathrm{http}: / /$ pmd.sourceforge.net/
}

Creative Research Groups of China No. 60821003, and the National Science Foundation of China No. 90718016.

\section{REFERENCES}

[1] J. Andersen and J. L. Lawall. Generic patch inference. In ASE, pages 337-346, 2008.

[2] J. Brunel, D. Doligez, R. R. Hansen, J. L. Lawall, and G. Muller. A foundation for flow-based program matching: using temporal logic and model checking. In $P O P L$, pages 114-126, 2009.

[3] J. Cheng, J. X. Yu, B. Ding, P. Yu, and H. Wang. Fast graph pattern matching. In ICDE, pages 913-922, 2008.

[4] T. Copeland. PMD Applied. Centennial Books, 2005.

[5] M. Gabel, L. Jiang, and Z. Su. Scalable detection of semantic clones. In ICSE, pages 321-330, 2008.

[6] E. Hill, L. Pollock, and K. Vijay-Shanker. Automatically capturing source code context of NL-queries for software maintenance and reuse. In ICSE, pages 232-242, 2009.

[7] R. Holmes and G. C. Murphy. Using structural context to recommend source code examples. In ICSE, pages 117-125, 2005.

[8] D. Hovemeyer and W. Pugh. Finding bugs is easy. SIGPLAN Not., 39(12):92-106, 2004.

[9] D. Janzen and K. De Volder. Navigating and querying code without getting lost. In AOSD, pages 178-187, 2003.

[10] L. Jiang, G. Misherghi, Z. Su, and S. Glondu. DECKARD: Scalable and accurate tree-based detection of code clones. In ICSE, pages 96-105, 2007.

[11] T. Kamiya, S. Kusumoto, and K. Inoue. CCFinder: a multilinguistic token-based code clone detection system for large scale source code. TSE, 28(7):654-670, 2002.

[12] Z. Li and Y. Zhou. PR-Miner: automatically extracting implicit programming rules and detecting violations in large software code. In ESEC/FSE, pages 306-315, 2005.

[13] D. Mandelin, L. Xu, R. Bodik, and D. Kimelman. Jungloid mining: Helping to navigate the API jungle. In PLDI, pages 48-61, 2005.

[14] M. Martin, B. Livshits, and M. S. Lam. Finding application errors and security flaws using PQL: A program query language. In OOPSLA, pages 365-383, 2005.

[15] A. Maule, W. Emmerich, and D. S. Rosenblum. Impact analysis of database schema changes. In ICSE, pages 451-460, 2008.

[16] S. Sinha, M. J. Harrold, and G. Rothermel. System-dependence-graph-based slicing of programs with arbitrary interprocedural control flow. In ICSE, pages 432-441, 1999.

[17] N. Tansalarak and K. T. Claypool. XSnippet: Mining for sample code. In OOPSLA, pages 413-430, 2006.

[18] S. Thummalapenta and T. Xie. ParseWeb: a programmer assistant for reusing open source code on the web. In ASE, pages 204-213, 2007.

[19] X. Wang, L. Zhang, T. Xie, H. Mei, and J. Sun. Locating need-to-translate constant strings for software internationalization. In ICSE, pages 353-363, 2009.

[20] M. Weiser. Program slicing. In ICSE, pages 439-449, 1981.

[21] W. Zhao, L. Zhang, Y. Liu, J. Sun, and F. Yang. SNIAFL: Towards a static noninteractive approach to feature location. TOSEM, 15(2):195-226, 2006.

[22] H. Zhong, T. Xie, L. Zhang, J. Pei, and H. Mei. MAPO: Mining and recommending API usage patterns. In ECOOP, pages 318-343, 2009. 